\title{
Active Galactic Nuclei in the Extreme Ultraviolet
}

\author{
ARIEH KÖNIGL \\ Department of Astronomy \& Astrophysics, University of Chicago, \\ 5640 S. Ellis Ave., Chicago, IL 60637, USA
}

\begin{abstract}
EUV observations could provide valuable and unique information about the nature of active galactic nuclei. I discuss their potential usefulness and review some of the main results obtained so far with the ROSAT WFC and the EUVE instruments. About three dozen sources, mostly Seyfert galaxies and BL Lacertae objects, have already been identified, and spectroscopic measurements of several of them have yielded a number of surprises. However, more observations are clearly needed to confirm some of the reported spectral features and to validate their interpretation.
\end{abstract}

\section{Introduction}

The extreme ultraviolet (EUV) wavelength interval (commonly defined to constitute the range $100-912 \AA$, or $13.6-124 \mathrm{eV}$ ) lies between the intensively studied FUV $(912-3000 \AA)$ and X-ray $(1-100 \AA)$ spectral regimes, but until recently it has not been subjected to systematic investigations. This lack of information has been particularly acute in the case of active galactic nuclei (AGNs), where the very ability to carry out successful EUV observations has been seriously questioned. This has stemmed from the fact that, for a Galactic H I column density $N_{\mathrm{G}}(\mathrm{HI})=1 \times 10^{20} \mathrm{~cm}^{-2}$, the interstellar transmission factor is 0.42 at $60 \AA$, but it is a factor of 10 smaller at $100 \AA$. Equivalently, each increment of $10^{20}$ in $N_{\mathrm{G}}(\mathrm{HI})$ contributes an optical depth of 3.2 at $100 \AA$. However, there are no known regions of the interstellar medium (ISM) that have $\left[N_{\mathrm{G}}(\mathrm{HI}) / 10^{20} \mathrm{~cm}^{-2}\right]<0.6$ (resp., 0.8) for positive (resp., negative) latitudes (e.g., Heiles 1991).

It has thus been gratifying to find out during the last few years that the "unobservable ultraviolet" could be measured after all, and that "clear windows to extragalactic space" do exist in the EUV wavelength regime. This opening up of the field of extragalactic EUV astronomy was made possible by the launch of the Wide Field Camera (WFC) on board ROSAT in 1990 and of the EUV-dedicated Extreme Ultraviolet Explorer with its three scanning telescopes and deep-survey spectrometer telescope in 1992 (see, e.g., Bowyer 1994 for a review). In this contribution, I give an overview of the results obtained so far by these observations and of their implications to the study of AGNs. After providing an interim tally of the identified objects, I discuss separately the two main classes of AGNs that have been found to exhibit significant EUV emission, namely Seyfert galaxies and BL Lacertae objects. I conclude with a brief discussion of future research prospects.

\section{EUV Observations of AGNs}

In accord with the anticipated effect of Galactic absorption, firm detections of AGNs have only been made in the shortest-wavelength bandpass of the ROSAT WFC (60-140 $\AA)$ and of the $E U V E$ photometers $(58-174 \AA)$. $\dagger$ In the same vein, those objects that

$\dagger$ In the few cases where EUV spectra have been measured (see $\$ 3$ and 4 below), the sources were found to be detectable only up to $\sim 110-120 \AA$. 
have been identified typically lie in the direction of Galactic neutral hydrogen "windows," along which the column density $N_{\mathrm{G}}(\mathrm{HI})$ does not exceed $\sim 1 \times 10^{20} \mathrm{~cm}^{-2}$ and for which the $\mathrm{He}$ II/H I ratio may also be favorable. In fact, the very detectability of an AGN may be used to place tight constraints on the helium abundance along the line of sight to the source. For example, by using the best-fit EUV energy spectral index $\alpha_{\text {euv }}=1.6$ obtained during the EUVE spectrometer calibration measurement of the BL Lac object PKS 2155304 together with the value $N_{\mathrm{G}}(\mathrm{HI})=1.36 \times 10^{20} \mathrm{~cm}^{-2}$ that had been determined from $21 \mathrm{~cm}$ emission measurements along the line of sight to this source, Fruscione et al. (1994) derived an upper limit of 0.16 on the ratio of the He II to $\mathrm{H}$ I column densities (assuming a negligible column of $\mathrm{He} \mathrm{I}$ in that direction).

The WFC Bright Source Catalog (Pounds et al. 1993) has identified 7 AGNs (4 Seyfertlike and 3 BL Lac objects [BLOs]), whereas EUVE observations have so far reported 7 AGNs (4 Seyferts [including Ton S 180, recently identified by Vennes et al. 1995] and 3 BL Lac objects) with a significance of $6 \sigma$ or better, and a total of 14 objects ( 8 Seyfert 1 galaxies, 5 BLOs, and 1 quasar [3C 273]) detected during the all-sky survey at $2.5 \sigma$ or better and lying within 60" of the AGN optical coordinates (Marshall et al. 1995b). Perhaps not surprisingly, the strongest EUV sources appear to be characterized by comparatively steep soft X-ray spectra. In fact, Marshall et al. (1995b) have argued that the relatively high number (compared to hard X-ray surveys) of detected BLOs is compatible with the steep X-ray spectra of the latter class of objects. $\dagger$ Although the $E U V E$ all-sky survey lists only 6 AGNs, additional sources continue to be identified by various other means, including pointed observations, serendipitous detections (McDonald et al. 1994), and a comparison between EUV and X-ray samples (Fruscione 1995). According to the most recent tally (given in the Second EUVE Source Catalog; Bowyer et al. 1995), the total number of EUV-detected AGNs now stands at 36.

\section{Radio-Quiet QSOs and Seyfert 1 Galaxies}

\subsection{Origin of the EUV-Soft X-ray Component}

EUV observations of radio-quiet QSOs and of the spectroscopically similar Seyfert 1 galaxies could potentially provide valuable clues to the nature of these sources. The peak power of radio-quiet QSOs is known to be emitted in the EUV range (several hundred $\AA$ ), and although Galactic absorption limits the useful data to $\lesssim 100 \AA$ (see $\S 1$ ), one might still expect to obtain useful information about the dominant AGN emission mechanism from successful EUV measurements. A related issue is the origin of the distinct soft X-ray component (representing a "soft excess" over an extrapolation of the ISM-absorbed hard $\mathrm{X}$-ray component) that is often measured in this class of AGNs. The exact properties of this component as well as its interpretation are still controversial. For instance, the first results of ROSAT Position Sensitive Proportional Counter (PSPC) observations of a complete sample of optically selected QSOs (Laor et al. 1994) have pointed to a spectral index $\alpha_{x}=1.50 \pm 0.40$ in the $0.2-2 \mathrm{keV}$ range that flattens by $\Delta \alpha_{x} \approx 0.5$ above $\sim 2 \mathrm{keV}$. This study has concluded that steep- $\alpha_{\mathrm{x}}$ sources are characterized by a weak

† It is interesting to note in this connection that, at least for non-BLO AGNs, the work of Mittaz et al. reported at this conference indicates that steep soft X-ray spectra, which are determined from models that include only a cold gas component along the line of sight to the source, are often associated with a low measured Galactic $\mathrm{H}$ I column. This correlation, which has been interpreted by Mittaz et al. in terms of a partially ionized absorber at the source, suggests that the apparent steepness of the spectrum may itself be a consequence of the low value of $N_{\mathrm{G}}(\mathrm{HI})$ and that the latter is, in fact, the primary factor that determines the EUV detectability of AGNs. 
hard component. A similar distribution of $\alpha_{\mathrm{x}}$ has been inferred also in other ROSAT observations of Seyfert 1 galaxies and radio-quiet QSOs (e.g., Turner et al. 1993; Walter \& Fink 1993; Fiore et al. 1994). These measurements have yielded a mean value of $\alpha_{\mathbf{x}}$ that is of the order of the mean value of $\alpha_{\text {ox }}$, the (rest frame) optical to X-ray (3000 $\AA-2 \mathrm{keV}$ ) spectral index. In contrast, previous studies (enumerated in Laor et al. 1994) by the Einstein and EXOSAT satellites have deduced steeper soft X-ray spectral slopes $\left(\alpha_{\mathrm{x}} \gtrsim 2\right)$ and have in some cases attributed the appearance of steep- $\alpha_{\mathrm{x}}$ sources to the presence of a strong soft X-ray component. The origin of the conflicting results could be either the better sensitivity of $R O S A T$ below $\sim 0.5 \mathrm{keV}$ or a PSPC calibration problem. EUV measurements could in principle strongly constrain the values of $\alpha_{\mathrm{x}}$ and of $\Delta \alpha_{\mathrm{x}}$ and thereby help determine the nature of the underlying emission mechanisms.

Lieu et al. (1995) have addressed this question using the data already at hand. They identified 6 AGNs for which $E U V E$ Lex/B count rates are available and for which ROSAT spectra that accurately define the soft X-ray spectral index have also been obtained. They found that the EUV count rates predicted by extrapolating the soft X-ray spectra are in fairly good agreement with the actual measurements over a significant dynamic range of count rates $(\sim 50)$ and intrinsic X-ray luminosities $\left(\sim 10^{4}\right)$. Although this sample clearly needs to be increased, the successful consistency check seems to provide strong support for the ROSAT measurements. The catch in this argument is that the soft X-ray spectra of the AGN sample utilized by Lieu et al. are considerably steeper than those of the average radio-quiet QSOs and Seyfert 1 galaxies measured by ROSAT; in fact, the mean value of $\alpha_{\mathbf{x}}$ for the 6 objects that they considered is 2.0 , which is consistent with the average values inferred by Einstein and EXOSAT (see, however, the previous footnote).

One can summarize the current state of affairs as follows. The apparent consistency between the ROSAT and EUVE observations supports the soft X-ray measurements carried out by the ROSAT PSPC. The implications of these measurements to the average radio-quiet AGNs are still not entirely clear, and recent studies (e.g., Walter et al. 1994; Fiore et al. 1995) have indicated that the optical through soft X-ray spectra of these sources cannot be explained in terms of a single simple model. Furthermore, it appears that the AGNs detected in the EUV have a particularly steep soft X-ray spectrum whose origin is also not yet fully understood. The data analyzed so far point to optically thick emission by a hot $\left(\sim 10^{6} \mathrm{~K}\right)$ gas, possibly associated with the innermost region of a nuclear accretion disk, and at least in some cases appear to be inconsistent with reprocessing of the observed hard X-ray component (e.g., Gondhalekar et al. 1994; Brandt et al. 1995; Marshall et al. 1995a).

\subsection{Probing the Warm Absorber}

The presence of a warm (i.e., partially ionized) absorbing gas along the line of sight to the continuum source has been inferred in a number of Seyfert 1 galaxies and QSOs from X-ray observations. Recent studies of objects like 3C 212 (Mathur 1994), 3C 351 (Mathur et al. 1994), and NGC 5548 (Mathur et al. 1995) have indicated that the same material is also responsible for the measured UV absorption, and that it can be identified with an outflowing (at a speed $\gtrsim 10^{3} \mathrm{~km} \mathrm{~s}^{-1}$ ) ionized gas of low density but high column density $\left(N_{\mathrm{H}} \gtrsim 10^{22} \mathrm{~cm}^{-2}\right)$ that is situated outside the Broad Emission Line Region (BELR). An analysis of the properties of this gas has led to the prediction that it could produce potentially detectable X-ray and UV emission features (Netzer 1993), and, in fact, an ASCA measurement of a $0.57 \mathrm{keV}$ feature (interpreted as an O VII emission line) that may be attributed to such a component has already been reported (George et al. 1995). 
It is thus natural to expect that EUV observations could also probe this gas and help to further constrain its properties.

Very recent work discussed at this conference has provided tantalizing suggestions that EUV emission lines which could be associated with the warm absorber have been observed in the Seyfert 1 galaxy NGC 5548 (paper presented by Kaastra \& Mewe; see also Kaastra et al. 1995) and possibly also Mrk 478 (paper presented by Liedahl et al.). The evidence for emission features in the EUVE spectra of these two sources has, however, been disputed by Marshall, who has interpreted the data in terms of pure continuum emission from an accretion disk (see also Marshall et al. 1995a). It is worth noting in this connection that both Kaastra et al. and Liedahl et al. have reached the rather unexpected conclusion that the emitting gas can be better described as being in collisional ionization equilibrium than in photoionization equilibrium (with an extra heat supply of the order of $10 \%$ of the bolometric luminosity indicated in the case of NGC 5548 in order to sustain the high inferred gas temperature). Thus, if the claims for the presence of EUV emission features in these objects and their explanation are corroborated, then the currently accepted interpretation of the warm absorber may need to be modified. In another presentation at this conference, Hwang et al. reported detecting emission features in the EUVE spectrum of the Seyfert 1 galaxy Mrk 279, but they found it difficult to obtain a good model fit to the data and, in any case, argued against an association of the features with a warm absorber. On the other hand, as was already alluded to in $\S 2$, Mittaz et al. reported indirect evidence for a warm absorber in a number of radio-quiet AGNs (including, in particular, those detected by the $E U V E$ ) that are characterized by a low value of $N_{\mathrm{G}}(\mathrm{HI})$. This rather confusing state of affairs might possibly be just a reflection of the extreme youth of the field of EUV spectroscopy, but it clearly argues for exercising great caution in interpreting the data and for the need for further observations.

\section{Blazars}

\subsection{Emission Signatures of a Relativistic Jet Component}

In contrast to Seyfert 1 galaxies and similar radio-quiet AGNs, whose emission over most wavelengths is evidently dominated by a thermal component, BLOs and other members of the "blazar" class of AGNs (notably high-polarization and optically violently variable QSOs) are distinguished by the dominance of a nonthermal emission component. This component, which is represented by a highly variable and often strongly polarized featureless continuum that extends from radio to $\mathrm{X}$-ray wavelengths, has been interpreted as synchrotron (and possibly also inverse-Compton) radiation originating in a relativistic jet (see, e.g., Königl 1989 for a review). In this picture, blazars are sources where the jet axis happens to be oriented at a small angle to the line of sight, so that its observed intensity is boosted by Doppler beaming. Certain AGNs, such as the bright nearby QSO 3C 273, show evidence for a blazar component that does not dominate the thermal emission, most likely because the jet is oriented at a comparatively large angle to the line of sight.

EUV observations of blazars could be useful for testing the relativistic jet interpretation and for helping to constrain the proposed models. They may be particularly helpful in the context of multiwavelength monitoring campaigns that attempt to determine the variability properties and the detailed spectra of these objects. Kafatos et al. have reported at this conference on preliminary results of such measurements for $3 \mathrm{C} 273$ and for the BLO Mrk 421. Significant EUV variability that is consistent with the relativistic beaming scenario has been detected on time scales ranging from hrs to weeks to months 
in multi-epoch observations of the BLO PKS 2155-304 (Marshall et al. 1993; Fruscione et al. 1994; Königl et al. 1995). The latter source has been the subject of extensive multiwavelength observations in the past. In particular, a recent campaign in 1991 November has detected $\gtrsim 20 \%$ soft X-ray flux variations occurring on a time scale of hrs and UV flux variations that followed the $\mathrm{X}$-ray changes with a lag of $\lesssim 3 \mathrm{hr}$, although a strict linkage was not maintained at all times (Urry et al. 1993; Brinkmann et al. 1994). These observations have been interpreted in terms of a shock wave propagating in the jet (Edelson et al. 1995). The soft X-ray measurements in the 1991 campaign have been carried out by ROSAT and included observations with the WFC that seemed to be consistent with the data acquired with the PSPC (Brinkmann et al. 1994). Further multiwavelength observations of this BLO that include the EUV spectral regime may help to clarify the UV-X-ray connection and test the shock interpretation (see also Marshall's contribution to these proceedings).

EUV measurements may also help to determine the precise contribution of the nonthermal component in radio-loud QSOs and the possible distinction between different types of BLOs. For example, Brunner et al. (1994) have recently presented ROSAT soft $\mathrm{X}$-ray spectra for a complete sample of core-dominated, flat-radio-spectrum AGNs, which included 8 quasars and 5 BLOs. They found that the quasars and BLOs were characterized by a mean value of $\left(\alpha_{\mathrm{ox}}-\alpha_{\mathrm{x}}\right)$ of $\sim 0.6$ and 0 , respectively, but that, in contrast to the quasars, the BLOs showed a relatively large dispersion $(\sigma \approx 0.5)$ about the mean (similar to that in $\alpha_{\mathbf{x}}$ ). Furthermore, the BLOs appeared to separate into two groups, one with $\alpha_{\mathrm{x}}<1.0$ and the other with $\alpha_{\mathrm{x}}>1.7$. As in the case of radio-quiet AGNs discussed in $\S 3.1$, EUV data could be used in conjunction with X-ray and optical/UV results to test the soft $\mathrm{X}$-ray measurements and help isolate the various spectral components that may be present. Another promising possibility, discussed at this conference by Marshall, is to attempt to measure EUV polarization (with the EUVE Deep Survey detector in an off-axis observing mode or with an optimized future telescope). This would be useful both for separating the thermal and nonthermal emission components and, once the relevant polarization mechanism is identified, for determining such important attributes of the emission region as its geometry and the local magnetic field distribution.

\subsection{EUV Spectroscopy of BL Lac Objects}

BLOs are distinguished by a weakness or absence of BELR emission. This has been attributed to the dearth of dense gas clouds (e.g., Guilbert et al. 1983), although the detectability of optical and UV emission lines is evidently also influenced by the strength of the underlying nonthermal continuum. Any spectral features that can be attributed to a circumnuclear gas are therefore of particular interest in these objects. One such feature, $\mathrm{a} \sim 0.6 \mathrm{keV}$ absorption trough or edge, was detected in PKS 2155-304 already in 1980 (Canizares \& Kruper 1984) and identified as an O VIII Ly $\alpha$ line originating in a mildly relativistic $(\sim 0.1 c)$ outflow at the redshift $(z=0.116$; Falomo et al. 1993) of the apparent host galaxy (Krolik et al. 1985). Tentative evidence for a similar feature has been reported also in other BLOs (Madejski et al. 1991). However, the 1980 Einstein Objective Grating Spectrometer (OGS) measurement of PKS 2155-304 appears to be formally inconsistent with a $B B X R T$ detection of a $\gtrsim 0.5 \mathrm{keV}$ absorption feature carried out 10 years later (Madejski et al. 1994), which has made the original identification of this feature problematic. This issue is related to the controversy over BLO distances. While these objects often appear in association with low-redshift $(z \lesssim 0.1)$ elliptical galaxies, it has been suggested that at least some of them might correspond to gravitationally lensed quasars located at higher redshifts (e.g., Ostriker \& Vietri 1985). If PKS 2155-304 in fact 
has a much higher redshift than that of the associated galaxy, then the identification of the OGS-detected feature with an O VIII Ly $\alpha$ line could not be correct.

Recent spectroscopic observations of this object with the $E U V E$ have shed important new light on these questions. The $E U V E$ has a state-of-the art spectrometer employing variable line-spaced gratings and equipped with advanced detectors that contain $1680 \times 1680$ independent resolution elements, are linear to $\lesssim 0.5 \%$, and have a quantum efficiency as high as $80 \%$ (e.g., Bowyer \& Malina 1991). In the on-axis observing mode, the shortest-wavelength channel encompasses $\sim 72-190 \AA$, and in the wavelength interval $72-100 \AA$ where most of the useful data have been obtained the spectral resolution ranges from $\sim 160$ to $\sim 200$. PKS 2155-304, which is one of the brightest X-ray selected BLOs, has turned out to be the brightest AGN in the EUV $\left(0.29 E U V E\right.$ cts s $\left.^{-1}\right)$, and has therefore been a prime candidate for $E U V E$ spectroscopic measurements.

Königl et al. (1995) carried out two EUVE observations of PKS 2155-304 during 1993 June and July with useful exposure times of $\sim 111$ and $\sim 157 \mathrm{ks}$, respectively. The source was detected in the $\sim 75-110 \AA$ range during both epochs, but the two spectra differed in detail, and the flux had increased by $\sim 50 \%$ between the two measurements (see $\S 4.1$ ). A power-law fit to the data has yielded an energy spectral index $\alpha_{\text {euv }} \approx 3-4$ for the measured Galactic H I column density and likely choices of the $\mathrm{He} \mathrm{I}$ and $\mathrm{He}$ II abundances. Such steep values are inconsistent with the soft X-ray spectral index of 1.65 measured by the ROSAT PSPC, which approximately corresponds also to the observed EUV to X-ray flux ratio. $\nmid$ Fitting a power law with $\alpha_{\text {euv }}=1.65$ to the EUV data implies strong absorption at the source between $\sim 75$ and $\sim 85 \AA$ (see Fig. 1). Königl et al. (1995) have argued that this absorption is not due to continuum opacity and demonstrated that it can be attributed, instead, to a superposition of Dopplersmeared absorption lines originating in high-velocity $(\leqslant 0.1 c)$, radially localized clouds of total column density $N_{\text {tot }} \approx 5 \times 10^{20} \mathrm{~cm}^{-2}$ that are ionized by the beamed continuum of the associated relativistic jet. They identified the lines as mostly L-shell transitions of $\mathrm{Mg}$ and $\mathrm{Ne}$ and $\mathrm{M}$-shell transitions of $\mathrm{Fe}$. The inferred ionization parameters and densities of the absorbing clouds are comparable to those of BELR clouds, but their velocities are an order of magnitude higher and their total column density is a factor $\gtrsim 10^{2}$ lower than the column of a standard BELR cloud. These values are consistent with the apparent dearth of BELR gas in BLOs as well as with Marshall et al.'s (1995b) conservative estimate (based on EUV data) that BLOs do not contain more than $10^{20}$ $\mathrm{cm}^{-2}$ of neutral hydrogen (since the deduced column density refers to ionized gas). It is, however, interesting to note that the cloud velocities and column densities obtained from this model fit are similar to those attributed to the Broad Absorption Line Region clouds detected in certain radio-quiet QSOs (e.g., Hamann et al. 1993).

The model used to fit the EUV spectrum also implies a pronounced O VII K $\alpha$ X-ray absorption feature at roughly the same energy as the feature detected in 1990 by $B B X R T$ (see discussion above). The predicted feature is broadened and blueshifted (relative to the cosmologically redshifted rest energy) by the motion of the absorbing gas and it dominates the soft X-ray spectrum. The maximum optical depth of the model feature is about an order of magnitude greater than that measured by $B B X R T$, although in making the comparison one should bear in mind that the $B B X R T$ and $E U V E$ observations were not simultaneous. For a given combination of $N_{\text {tot }}$ and of the $\mathrm{Mg}, \mathrm{Ne}$, and Fe abundances

$\dagger$ Given the various observational uncertainties, this value of $\alpha_{\mathbf{x}}$ is also compatible with the EUV measurements by the ROSAT WFC (Brinkmann et al. 1994), and it thus appears that any break to the flatter $(\alpha<1)$ spectrum recorded by the IUE (Urry et al. 1993) does not occur at wavelengths shorter than $\sim 124 \AA$. 
PKS 2155-304
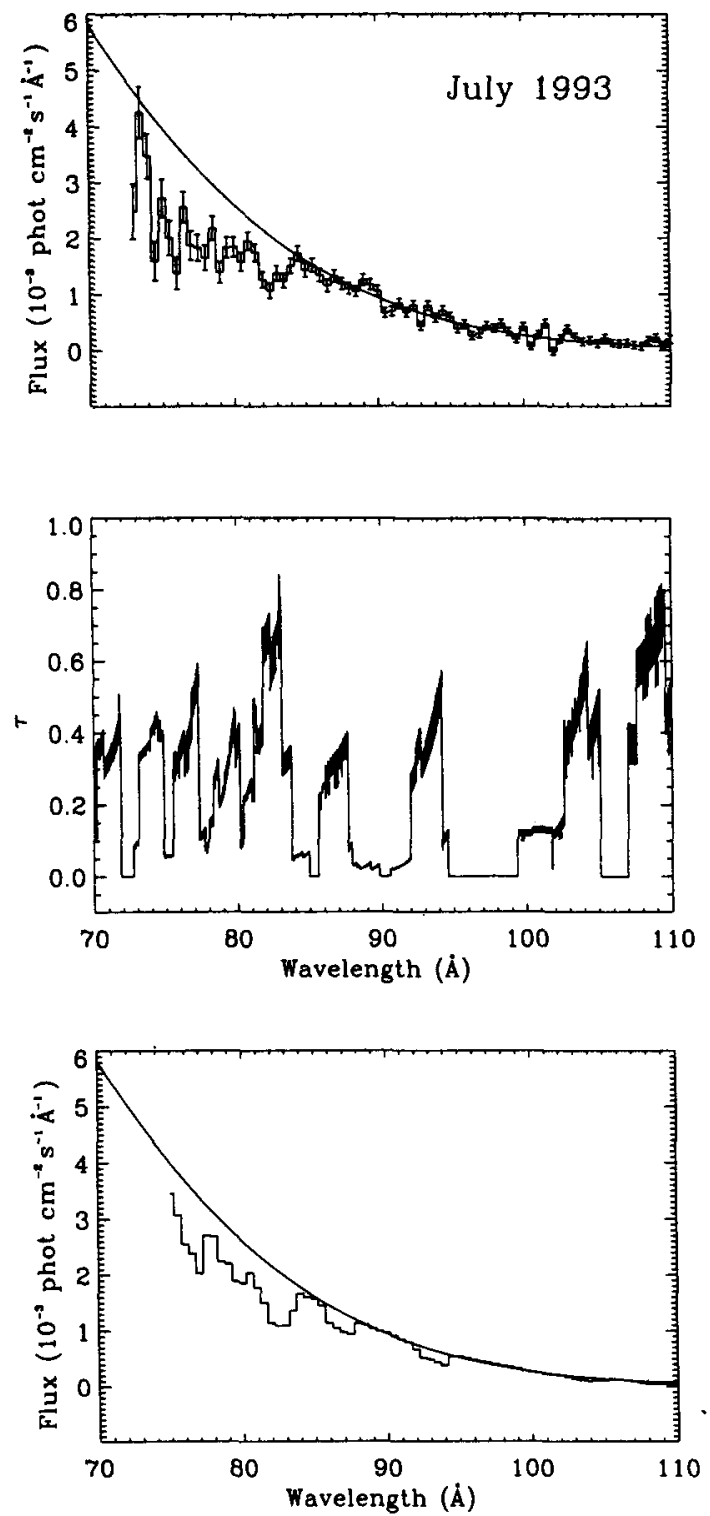

FIgURE 1. The top panel displays the background-subtracted spectrum (shown with $1 \sigma$ error bars) for the 1993 July EUVE observations of PKS 2155-304 (from Königl et al. 1995). The solid line represents a best-fit (in the range $85-110 \AA$ ) power-law continuum of energy spectral index $\alpha_{\text {euv }}=1.65$, modified by Galactic absorption. The middle panel presents the EUV line opacities calculated for a clumped outflow model, and the bottom panel shows the fit to the above data obtained by incorporating the effect of the outflow line and continuum opacities on the $\alpha_{\text {euv }}=1.65$ power-law continuum (represented again by the solid curve). 
that reproduces the EUV data, the predicted optical depth of the X-ray feature can be adjusted by varying the relative abundance of oxygen (which, in turn, does not affect the EUV spectrum below $\sim 110 \AA$ ). It can be verified that the alternative interpretation of the $B B X R T$ observations in terms of an oxygen $\mathrm{K}$ edge at a redshift in the range $0.18-0.42$ (Madejski et al. 1994) is inconsistent with the $E U V E$ observations, basically because of the large values of $N_{\text {tot }}\left(\gtrsim 5 \times 10^{21} \mathrm{~cm}^{-2}\right)$ that it involves. The EUV and X-ray data together thus reinforce the identification of this BLO with a galaxy at $z=0.116$.

The interpretation of the EUV and X-ray spectra of PKS 2155-304 in terms of highvelocity clouds that occupy a relatively narrow range of distances from the central object suggests a possible explanation of the apparent discrepancy between the $\mathrm{X}$-ray spectra obtained by Einstein and $B B X R T$. In this picture, the difference between the two measurements could be attributed to the absorbing clouds observed by Einstein having crossed our line of sight closer to the continuum source than the clouds observed a decade later, so that their dominant oxygen ion was O VIII rather than O VII. These two groups of clouds might conceivably be associated with a single global event: for example, they could be related to a disk instability wherein a propagating front induces an outflow at progressively larger radii (a situation possibly resembling FU Orionis outbursts in pre-main-sequence stars; e.g., Calvet et al. 1993, Bell \& Lin 1994). Coordinated EUV and X-ray observations could test whether the long-term X-ray spectral variability is correlated with changes in the EUV spectrum, as predicted by this scenario, and whether such variations indeed exhibit a systematic trend in the underlying ionization and velocity structures.

\section{Conclusion}

The field of EUV astronomy, particularly in regard to AGNs, is still in its infancy. These are exhilarating times, with new discoveries following each other in close succession, with cherished beliefs appearing to be in danger of imminent overhaul, and with conflicting claims and confusion clouding the scene. This brief review was merely meant to provide a synopsis of the recent developments in this field and of their relevance to AGN research rather than an evaluation of already well established results. The promising directions for future studies, predicated on the assumption that EUV telescopes will continue to be built and operated, can be summarized as follows.

- Radio-quiet QSOs and Seyfert 1 galaxies

(a) Increase the data base for comparing EUV flux measurements with soft X-ray spectral extrapolations ( $\$ 3.1$ ).

(b) Search for the signatures of the warm absorber $(\S 3.2)$.

- Blazars

(a) Probe for signatures of beamed nonthermal continuum ( $\$ 4.1$ ).

(b) Attempt to measure the EUV spectra of other BLOs besides PKS 2155-304-e.g., Mrk 421.

(c) Carry out off-axis EUVE observations of PKS 2155-304 (and other EUV-bright AGNs) with the aim of broadening the useful spectral range (down to $\sim 64 \AA$ ). $\dagger$

(d) Monitor the spectral variability of bright BLOs for EUV-X-ray correlations and for systematic trends in the inferred ionization and velocity structures (§ 4.2).

I am grateful to my collaborators in the EUVE observations project, John Kartje, Stuart Bowyer, Steven Kahn, and Chorng-Yuan Hwang, for their valuable input into this

$\dagger$ Such observations have already been attempted; e.g., in the case of the Seyfert 1 galaxy Mrk 478 (Marshall et al. 1995a). 
work. This research was supported in part by NASA grants NAG 5-2265 and NAGW1636 .

\section{REFERENCES}

BELL, K. R., \& LIN, D. N. C. 1994, ApJ, 427, 987

BOWYER, S. 1994, Science, 263, 55

BOWYER, S., ET AL. 1995, ApJS, in press

BowYer, S., \& MALINA, R. F. 1991, in Extreme Ultraviolet Astronomy, ed. R. F. Malina \& S. Bowyer, New York: Pergamon, 397

Brandt, W. N., Pounds, K. A., \& Fink, H. 1995, MNRAS, 273, L47

BrinkmanN, W., ET AL. 1994, A\&A, 288, 433

Brunner, H., Lamer, G., Worrall, D. M., \& Staubert, R. 1994, A\&A, 287, 436

Calvet, N., Hartmann, L., \& Kenyon, S. J. 1993, ApJ402, 623

Canizares, C. R., \& Kruper, J. 1984, ApJ, 278, L99

EDELSON, R., ET AL. 1995, ApJ, 438, 120

Falomo, R., Pesce, J. E., \& Treves, A. 1993, ApJ, 411, L63

Fiore, F., Elvis, M., McDowell, J., SiemiginowskA, A., \& Wilkes, B. J. 1994, ApJ, 431, 515

Fiore, F., Elvis, M., Siemiginowska, A., Wilkes, B., McDowell, J., \& Mathur, S. 1995, ApJ, in press

Fruscione, A. 1995, ApJ, submitted

Fruscione, A., Bowyer, S., Königl, A., \& KaHN, S. M. 1994, ApJ, 422, L55

George, I. M., Turner, T. J., \& Netzer, H. 1995, ApJ, 438, L67

Gondhalekar, P. M., Kellett, B. J., Pounds, K. A., Matthews, L., \& Quenby, J. J. 1994, MNRAS, 268, 973

Guilbert, P. W., Fabian, A. C., \& McCray, R. 1983, ApJ, 266, 466

HamanN, F., Korista, K. T., \& Morris, S. L. 1993, ApJ, 415, 541

HeILES, C. 1991, in Extreme Ultraviolet Astronomy, ed. R. F. Malina \& S. Bowyer, New York: Pergamon, 318

KaAstra, J. S., Roos, N., \& Mewe, R. 1995, A\&A, in press

KönIGL, A. 1989, in BL Lac Objects, ed. L. Maraschi, T. Maccacaro, \& M.-H. Ulrich, Springer, 321.

Königl, A., Kartje, J. F., Bowyer, S., KahN, S. M., \& Hwang, C. -Y. 1995, ApJ, 446, 598

Krolik, J. H., Kallman, T. R., Fabian, A. C., \& Rees, M. J. 1985, ApJ, 295, 104

LaOR, A., Fiore, F., Elvis, M., Wilkes, B. J., \& McDowell, J. C. 1994, ApJ, 435, 611

Leu, R., Mittaz, J., Bowyer, S., Lewis, J., \& Hwang, C. -Y. 1995, Adv. Space Res., $16(3), 81$

MADEJSKI, G. M., ET AL. 1994, preprint

Madejski, G. M., Mushotzky, R. F., Weaver, K. A., Arnaud, K. A., \& Urry, C. M. 1991, ApJ, 370, 198

Marshall, H. L., Carone, T. E., \& Fruscione, A. 1993, ApJ, 414, L53

Marshall, H. L., Carone, T. E., Shull, J. M., Malkan, M. A., \& Elvis, M. 1995a, ApJ, submitted

Marshall, H. L., Fruscione, A., \& Carone, T. E. 1995b, ApJ, 439, 90

Mathur, S. 1994, ApJ, 431, L75

MathuR, S., Elvis, M., \& Wilkes, B. 1995, ApJ, in press

Mathur, S., Wilkes, B., Elvis, M., \& Fiore, F. 1994, ApJ, 434, 493 
A. KöNIGL

MCDonald, K., ET AL. 1994, AJ, 108, 1843

NeTZER, H. 1993, ApJ, 411, 594

Ostriker, J. P., \& VIETri, M. 1985, Nature, 318, 446

Pounds, K. A., ET AL. 1993, MNRAS, 260, 77

Turner, T. J., George, I. M., \& Mushotzky, R. F. 1993, ApJ, 412, 72

UrRY, C. M., et AL. 1993, ApJ, 414, 614

Vennes, S., Polomsky, E., Bowyer, S., \& Thorstensen, J. R. 1995, ApJ, submitted

WALTER, R., ET AL. 1994, A\&A, 285, 119

WALTER, R., \& FinK, H. H. 1993, A\&A, 274, 105 